\title{
Validación de un Cuestionario de Evaluación de la Ética Profesional Docente Universitaria
}

\section{Validation of a University Teaching Professional Ethics Evaluation Questionnaire}

\author{
Alma Delia Torquemada González ${ }^{1,{ }^{*}}$ y Javier Loredo Enríquez ${ }^{2}$ \\ ${ }^{1}$ Universidad Autónoma del Estado de Hidalgo, México \\ ${ }^{2}$ Universidad Iberoamericana, CDMX
}

\author{
DESCRIPTORES: \\ Evaluación docente \\ Ética docente \\ Ética profesional \\ Docencia universitaria
}

\begin{abstract}
RESUMEN:
La ética profesional como rasgo característico de una docencia de excelencia es reconocida desde la investigación educativa; no obstante, su evaluación en el desempeño docente es apenas visible en el contexto universitario. Este trabajo tiene como finalidad presentar el proceso de construcción y validación de un cuestionario de opinión docente orientado a la identificación de las características que definen la ética profesional en la docencia universitaria y su evaluación en el desempeño docente. Metodológicamente se describe el proceso de construcción de las dimensiones teóricas del instrumento a través de un análisis de contenido. Posteriormente, se explica el proceso de validación del cuestionario mediante una entrevista cognitiva. Como resultado se presenta el cuestionario final, el cual asume una escala Likert conformada por 49 reactivos, organizados en cuatro dimensiones y seis ejes analíticos. Se concluye señalando que el cuestionario sitúa a la ética profesional como una dimensión específica dentro de los procesos de evaluación docente, reconociendo la participación de los profesores universitarios en la valoración del propio desempeño ético. Analizar el profesionalismo docente desde la perspectiva de sus propios actores, permitirá generar procesos formativos orientados a la búsqueda del compromiso con la docencia favoreciendo una evaluación docente integral.
\end{abstract}

\section{KEYWORDS:}

Teaching Evaluation Teaching ethics

Professional ethics

University teaching

\begin{abstract}
:
Professional ethics as a characteristic feature of excellent teaching is recognized by educational research, however, its evaluation in teaching performance is barely visible in the university context. The purpose of this paper is to present the process of construction and validation of a teacher's opinion questionnaire aimed at identifying the characteristics that define professional ethics in university teaching and their evaluation in teaching performance. Methodologically, the process of construction of the theoretical dimensions of the instrument is described through a content analysis. Subsequently, the validation process of the questionnaire is explained through a cognitive interview. As a result, the final questionnaire is presented, which assumes a Likert scale made up of 49 items, organized in four dimensions and six analytical axes. It is concluded by noting that the questionnaire places professional ethics as a specific dimension within the teacher evaluation processes, recognizing the participation of university professors in the assessment of their own ethical performance. Analyzing teaching professionalism from the perspective of its own actors, will allow to generate training processes oriented towards the search for commitment to teaching, favoring a comprehensive teaching evaluation.
\end{abstract}

CÓMO CITAR:

Torquemada, A. D. y Loredo, J. (2021). Validación de un cuestionario de evaluación de la ética profesional docente universitaria. Revista Iberoamericana de Evaluación Educativa, 14(1), 101-114.

https://doi.org/10.15366/riee2021.14.1.006

*Contacto: almatorquemada@gmail.com

ISSN: 1989-0397

https://revistas.uam.es/riee 


\section{Introducción}

Hoy en día, la educación superior y en particular la docencia enfrenta un desafío ético al esperarse un mayor impacto en la formación integral de los jóvenes y no solamente un proceso de instrucción para la inserción al ámbito laboral; esto requiere no sólo del desarrollo de programas de formación docente permanente, sino además de un mecanismo de evaluación más completo que permita retroalimentar la dimensión ética del acto de enseñar, o mejor dicho del acto de educar en el nivel superior (Chávez y Benavides, 2011; García et al., 2010). Por este motivo, es necesario entender el verdadero sentido de la evaluación docente como mecanismo de mejora de la práctica desde la ética profesional.

Si bien, la evaluación docente en el nivel superior constituye uno de los mayores tópicos educativos de interés en México, su impacto en términos de mejora de la práctica aún es débil. Como señalan Rueda (2010) y Coloma (2010), la evaluación se hace necesaria para mejorar el desempeño académico, planteándose con un fin formativo asociado a un proceso de mejora permanente que incida en el perfeccionamiento no sólo de sus prácticas de enseñanza, sino también de su personalidad, transformando así sus capacidades humanas.

En este sentido, la evaluación docente con fines de mejora en el contexto universitario es una tarea pendiente. Al respecto, Iglesias y Loredo (2017) afirman que una evaluación carente de significado para el profesor no favorece el desarrollo profesional de la docencia. Una evaluación será reconocida y aceptada por los profesores en la medida en que se tomen en cuenta sus opiniones y experiencias; esto hará que se sientan escuchados y en consecuencia valorados, propiciando con ello un compromiso hacia la mejora de su práctica.

Sería deseable que en toda evaluación del desempeño docente orientado al perfeccionamiento de la práctica se valoraran determinadas competencias como la crítica reflexiva sobre su propio rol como profesor y sobre el ejercicio de la disciplina en el ámbito laboral en la que se está formando a sus estudiantes; o bien, cuestionarse su vocación hacia la enseñanza, al mismo tiempo que puedan observarse con cuidado determinados rasgos del carácter que impactan en la formación estudiantil y no privilegiar únicamente competencias didáctico-pedagógicas.

Bajo este marco de ideas, hablar de una evaluación docente ética no es una tarea sencilla, pues se precisa de una sólida formación en el docente en cuanto a actitudes, aptitudes, competencias, conocimientos y voluntad se refiere, para poder desarrollarla correctamente (Hortal, 2000; Sanz, 2019). Este desafío solamente surge de aquellos docentes comprometidos, dispuestos a cambiar el curso de las cosas y situarse siempre del lado del estudiante, intentando favorecer su desarrollo.

En congruencia con Murillo (2018), la cuestión primordial hoy en día no es seguir discutiendo si debe o no desarrollarse la evaluación docente en las universidades, ni tampoco cuestionarse sobre los medios de evaluación más efectivos; el debate debe centrarse en saber cómo pueden realizarse evaluaciones cada vez más justas y equitativas que les permitan a los docentes tener información creíble, transparente, práctica y útil con una orientación hacia la mejora de los procesos educativos y de su propio rol como formadores de futuros profesionistas; que sea además contextualizada y genere el compromiso docente.

\section{Fundamentación teórica}

La ética profesional como rasgo distintivo de una docencia de calidad en el nivel superior ha cobrado especial interés desde el campo de la investigación educativa; al respecto, diversas investigaciones ponen en tela de juicio el actuar docente en el contexto universitario (Campo-Cabal, 2012; Finkler et al., 2012; Hirsch, 2011; Rancich et al., 2015; Torres, 2006), reportando conductas no éticas en el ejercicio docente y su impacto en la formación integral del estudiante en diferentes ámbitos de formación disciplinar.

Situaciones tales como la falta de atención e interés por las problemáticas particulares del estudiante, relaciones negativas con los estudiantes, maltrato al interior del aula, hostigamiento y acoso sexual, discriminación hacia el estudiante, subjetividad en la evaluación de los aprendizajes, temor a ser castigados con bajas calificaciones, un trato despectivo, o bien, situaciones competitivas entre estudiantes que tienen que ver con la defensa de las normas o los derechos individuales. 
Dichas problemáticas afectan el estado emocional del estudiante, el ambiente de aprendizaje y por supuesto, la motivación hacia el ejercicio de la profesión, con el riesgo de que dichas conductas sean reproducidas después por el estudiante en el campo laboral. Estas situaciones son cada vez más frecuentes al interior de las universidades, generando la necesidad de debatir sobre principios universales como equidad y respeto.

Por este motivo, en el contexto mexicano, diversas instituciones de nivel superior tanto del sector público como privado han implementado durante los últimos años, protocolos para la atención y prevención de la violencia de género en sus instituciones, desarrollando mecanismos de actuación y un marco normativo.

La generación de protocolos sobre ética es el resultado de una de las metas más importantes de la agenda educativa internacional al 2030 propuesta por la UNESCO (2016), en la que se contempla como reto combatir la desigualdad de género, la inequidad y la exclusión. La desigualdad de género es un grave problema para el desarrollo humano presente en todos los ámbitos sociales; por ello, se busca que la universidad, como espacio para la producción y reproducción de conocimiento asuma la responsabilidad de empoderar a la igualdad de género, factor clave para el desarrollo de un país. Para asumir dicha responsabilidad la universidad implementa una serie de acciones, tales como: generar ambientes donde los estudiantes puedan ejercer sus derechos y al mismo tiempo desarrollar sus identidades individuales y de grupo, así como promover prácticas de formación docente, con las que la igualdad de género sea parte de su misión formativa (UNESCO, 2016).

Por lo tanto, se espera que la educación superior forme profesionales y ciudadanos que en los diferentes contextos aporten un ambiente de respeto, de derechos entre hombres y mujeres, siendo protagonistas en la defensa hacia los demás, garantizando paz, respeto y solidaridad. En la medida en que la universidad forme profesionales empoderados a nivel intelectual, académico, científico y humanístico será posible impulsar una sociedad más justa, equitativa y solidaria (Mayorga, 2018).

De aquí que un objetivo fundamental que persiguen las universidades sea promover un cambio ético en la educación, incorporando en sus sistemas axiológicos el respeto a la diversidad. Para avanzar en esta meta, los docentes son actores centrales, desempeñando un rol clave en el proceso de transformación social (UNESCO, 2016). Difícilmente se podrá aspirar a una educación para la justicia social si no se cuenta con mecanismos de evaluación que sean congruentes con estos principios. La evaluación educativa centrada en principios de justicia social requiere analizar las condiciones sociales de los individuos, su contexto cultural y las necesidades reales que obstaculizan su desarrollo.

En este sentido, una evaluación de esta naturaleza tendría que verificar la universalidad e igualdad en el acceso a la educación; dar cuenta de la heterogeneidad social y cultural de los estudiantes y las escuelas; demostrar los procesos reales de enseñanza y aprendizaje que tienen lugar en el aula reconociendo la dinámica escolar asegurando que se cumpla con el principio de trato equitativo y diferenciado; evaluar la existencia de normas de convivencia justas y consensuadas con la comunidad y valorar tanto la actitud como el compromiso de docentes y estudiantes.

Tal y como señalan Nava (2011) y Díaz-Barriga y otros (2016) los profesores en el ámbito universitario gozan de un poder en el aula por ser de mayor jerarquía y tener un dominio de la disciplina, permitiéndoles decidir lo que van a hacer y cómo lo van a hacer, manteniendo un cierto margen de autonomía en su pensar, hacer y actuar. Así, bajo un enfoque humanista construir una educación para la justicia social requiere contar con una evaluación igualmente justa, equitativa, inclusiva y participativa que priorice la reflexión sobre la mejora del propio desempeño (Murillo et al., 2011). En esta perspectiva se inserta la evaluación docente de la ética profesional.

Por lo tanto, el proceso de enseñanza y aprendizaje solo puede cambiar si los docentes identifican las necesidades de sus estudiantes y si se convierten en modelo a seguir en la promoción de la igualdad de género y respeto. Por este motivo, la actitud docente juega un papel determinante en la creación de un ambiente apropiado de clase, libre de prejuicios de género, capaz de manejar cualquier situación en la que haya diferencias, ya sean de género o de cualquier índole (racial, física, ética). Son los maestros quienes deben tener conciencia de sus propias creencias y comportamientos con los alumnos para lograr que la igualdad de género sea una realidad en todos los entornos de aprendizaje (relaciones profesor-alumno, actividades, tareas y evaluación); de ahí que la ética profesional de la docencia sea un factor determinante para la generación de ciudadanos más justos y comprometidos con su sociedad. 
Por ello, resulta esencial la instauración de iniciativas sobre procesos de evaluación formativa del profesorado orientadas a elevar la calidad docente dentro de las universidades, las cuales podrían generar oportunidades para reflexionar y acordar las características que dicha actividad debe cubrir en función del contexto que lo caracteriza; esto implica repensar la evaluación docente desde una visión humanista.

Asumir una postura humanista en el contexto educativo significa privilegiar el respeto a los derechos de la persona, la consideración de sus sentimientos y la preocupación por no provocarle ningún daño; es decir considerar al docente como un ser humano, una persona con expectativas e intereses y emocionalmente involucrada antes que un empleado o un mero profesionista (Loredo, 2000).

De esta manera, la preocupación central de un proceso de evaluación de la docencia debe ser la preservación de la dignidad, la autoestima y la individualidad de los profesores, lo cual será posible si se asume un modelo de desarrollo o perfeccionamiento docente permanente. El ejercicio docente requiere de una evaluación que le permita retroalimentar su práctica y que esté vinculada a procesos formativos de carácter ético.

Al menos en el contexto mexicano, diariamente ingresan profesionistas para desempeñarse como docentes universitarios sin tener competencias específicas para ello. Pese a que reciben cursos de actualización y/o capacitación docente, carecen de habilidades para la solución de conflictos profesionales relacionados con la ética, o bien, prestan poca atención a los problemas ético-morales durante su práctica pedagógica. La ausencia de un proceso de concientización sobre el propio comportamiento del profesor, provoca que se transgreda la ética durante el ejercicio docente, por ello, pensar en el impacto de sus acciones en la formación de futuros profesionistas es una tarea pendiente al interior de las universidades.

De acuerdo con Cortina (2017), la ética profesional en la docencia no ha sido discutida con profundidad desde las políticas de calidad educativa en las universidades, si bien, existen códigos deontológicos que impulsan la identificación con los ideales universitarios, aún hace falta discutir la práctica de la ética en el ejercicio cotidiano de la docencia. Hasta el momento, únicamente es posible observar un esfuerzo por delimitar valores asociados a la ética profesional en función de la visión y misión de las instituciones educativas que se traducen en perfiles profesionales del docente ideal. Es preciso cuestionar y reflexionar lo que representa una docencia ética en el actuar cotidiano de la vida académica y cómo es valorado dicho actuar en las evaluaciones del desempeño docente.

En concordancia con Hortal (2002) y García y otros (2011), se ha perdido una valiosa oportunidad para incorporar de forma más visible una de las dimensiones que caracterizan a un buen profesional: la dimensión ética. Desde la postura de los autores, en todo ámbito laboral, los problemas éticos están presentes en el desempeño profesional, de ahí que la ética sea la gran olvidada en todas las reformas y la más importante porque se buscan profesionales benevolentes, preocupados por el mundo, poniendo su formación y su conocimiento al servicio de una sociedad mejor, más justa, más equitativa, que defienda la igualdad de oportunidades.

La ética profesional de la docencia se apega a una serie de principios que marcan su profesionalización (Hortal, 2000), los cuales son: beneficencia que implica buenas prácticas docentes encaminadas al beneficio de otras personas, en este caso los universitarios; la no maleficencia, es decir una actitud docente que no daña a sus alumnos, ni a sus familias; principio de autonomía que significa el desempeño laboral del docente en un marco de independencia y libertad, lo cual le permite intervenir de una manera ética. El principio de justicia, que guía a un docente a cumplir con sus deberes y obligaciones; explicar correctamente contenidos, actualizarse y preocuparse por el bienestar de los estudiantes, haciendo un seguimiento adecuado de su proceso educativo. Estos principios éticos están presentes en la interacción del docente con el estudiante y son evidencia del compromiso que asume la docencia con las familias de sus estudiantes y con la comunidad.

Los planteamientos anteriores permiten reconocer la presencia de la ética profesional en el actuar cotidiano del profesor. Al respecto, diversos autores (Camps, 2008; Chávez, 2019; Chávez y Benavides, 2011; Hirsch, 2019; Sanz, 2019) manifiestan que durante las prácticas de enseñanza y aprendizaje, los docentes ponen en marcha una serie de principios éticos implícitos en sus interacciones cotidianas que guían los procesos de enseñanza y aprendizaje con sus alumnos, siendo éstos: el diálogo, la comunicación, la empatía, la escucha activa, la armonía en el aula, el apoyo e interés ante problemas personales que afectan 
el desempeño académico; respeto, trato digno, el compromiso con la docencia y la responsabilidad que se asume con la formación del estudiante.

En este sentido, los aspectos personales del profesor, referidos al tipo de vínculo que establece con el alumno son aspectos a reflexionar cuando se habla de una evaluación de la ética docente. Desde la perspectiva de la evaluación formativa, la importancia de evaluar la función docente es lograr el mejoramiento del profesor como persona y docente, así como de todos los componentes del proceso de enseñanza-aprendizaje. El impacto de la evaluación formativa se reflejará en el desarrollo social y emocional de los alumnos, en la adquisición de conocimientos, en la renovación metodológica y de materiales educativos, así como también en la cooperación dentro y fuera del aula y en el autodesarrollo (Arbesú et al., 2011).

Concebir procesos de evaluación formativa en torno a la ética profesional requiere del análisis situacional de la práctica docente y del reconocimiento del contexto social, político e histórico en el que se encuentran insertas las instituciones de nivel superior hoy en día.

Investigadores como Sanz (2019), Coloma (2010) y Chávez (2019) manifiestan que en los instrumentos de evaluación se tocan algunos rasgos muy genéricos sobre la actitud del docente hacia el alumno, denominados competencias interpersonales, socioemocionales, o bien, se mencionan como complemento de otros rasgos pedagógicos; en realidad se carece de una delimitación precisa sobre la ética profesional docente, así como de criterios específicos para su evaluación.

A partir de los argumentos anteriores, la problemática de interés se inserta en el ámbito de la evaluación de la ética profesional de la docencia universitaria, específicamente, en la delimitación de aquellas acciones éticas que ponen en juego los docentes durante el proceso de enseñanza-aprendizaje con sus estudiantes y su inserción como una dimensión específica dentro de los procesos de evaluación de su desempeño, esto a través de la construcción de un cuestionario dirigido a docentes universitarios.

Esto forma parte de un proyecto de investigación más amplio centrado en profundizar en la necesidad de una evaluación docente integral que sitúe la reflexión sobre la ética profesional docente desde las experiencias de sus principales involucrados: los docentes. Cabe aclarar que dicha investigación comprende el diseño y validación de dos instrumentos, uno cualitativo y otro cuantitavo. El instrumento cualitativo se refiere a una guía de entrevista con la cual se desarrollarán grupos de discusión con docentes sobre sus experiencias en torno a la presencia de la ética profesional en las interacciones cotidianas con sus estudiantes. El instrumento cuantitativo corresponde al cuestionario docente orientado a indagar aquellos rasgos que caracterizan mejor la ética profesional en las prácticas de enseñanza en el nivel superior y su inserción en la evaluación del desempeño docente. La combinación de instrumentos cualitativos y cuantitavos permitirá comprender la complejidad de la evaluación de la ética profesional docente en la diversidad de contextos, valorar su práctica docente como campo de saber y ejercicio profesional.

Para fines del presente artículo se presenta el proceso de construcción del instrumento cuantitativo, es decir el cuestionario, así como su validación a través de una entrevista cognitiva realizada con 6 docentes de distintas universidades mexicanas públicas y privadas. El cuestionario como una primera vía de exploración de las opiniones docentes permitirá identificar la ética profesional docente, sus rasgos, prácticas cotidianas en la interacción de profesores y estudiantes en el nivel superior, así como su presencia dentro de los procesos de evaluación del desempeño docente. Dicho instrumento será aplicado en un segundo momento a docentes universitarios en sus diferentes disciplinas provenientes de la Universidad Autónoma del Estado de Hidalgo (UAEH), México.

\section{Método}

\subsection{Construcción del cuestionario}

Para la construcción de las dimensiones del cuestionario, se partió de una revisión de la literatura con base en dos aproximaciones analíticas: una teórica y otra investigativa. En la primera aproximación, se identificaron aquellos elementos teóricos que permitieron conceptualizar la ética profesional docente. En la segunda, se revisaron un total de 64 investigaciones del contexto nacional e internacional, de las cuales se eligieron aquellos estudios que dieran cuenta de las competencias y/o rasgos de ética profesio- 
nal docente que mejor caracterizan la ética docente en el nivel superior. De igual forma, se identificaron instrumentos y procesos orientados a la evaluación de la ética profesional docente. Esta fundamentación teórica se observa en el Cuadro 1.

Cuadro 1

Fundamentación teórica en la construcción del cuestionario

Fundamentos teóricos

Surgio de las aportaciones teóricas sobre ética de Gómez (2014); Ruiz (2016); Cortina (2017); Lévinas (2001); Jaramillo y Aguirre (2010); Camps (1996; 2008) y ética profesional de Hortal (2002); López-Calva y Gómez (2017).
Investigaciones en el

contexto mexicano e internacional
Se obtuvo de los hallazgos de las investigaciones más representativas en el contexto mexicano, tales como: Hirsch (2010a, 2010b, 2019), Chávez, (2019), Medina, et. al. (2010), Chávez y Benavides (2011), Tapia (2019), Águila, et. al. (2014), García-Cabrero, et. al. (2014), López-Calva, et. al. (2017) y un estado del conocimiento editado por COMIE (Hirsch y Yurén, 2013); así como también de las investigaciones del contexto internacional centrados en la evaluación de la ética profesional docente, como son: García, et. al. (2006), Solano y Campos (2014), Villarroel y Bruna (2017), Salazar, et. al. (2018), Serrano, et. al. (2018), Safari y Yoosefpour (2018) y Navia (2019).

Nota. Elaboración propia.

A partir de un análisis de contenido (Álvarez-Gayou, 2019; Flick, 2014) sobre los elementos teóricos y los hallazgos más relevantes de las investigaciones en torno a la ética profesional docente se procedió a definir las 4 dimensiones teóricas y los ejes analíticos que los conforman, los cuales permitieron a su vez generar los reactivos para el cuestionario de evaluación de la ética profesional docente. Este proceso de construcción conceptual se presenta en el Cuadro 2.

Cuadro 2

Dimensiones y ejes analíticos del cuestionario de evaluación de la ética profesional docente

\begin{tabular}{|c|c|c|}
\hline Dimensiones & Conceptualización & Ejes analíticos \\
\hline $\begin{array}{l}\text { La ética en la docencia univer- } \\
\text { sitaria }\end{array}$ & $\begin{array}{l}\text { Se entiende como la presen- } \\
\text { cia de la ética profesional en } \\
\text { la docencia universitaria, su } \\
\text { relevancia en el desempeño } \\
\text { docente y formativo. }\end{array}$ & $\begin{array}{l}\text { 1) La profesión docente: Son los aspectos relativos } \\
\text { a la vocación docente que tienen lugar tanto en } \\
\text { el desarrollo profesional en la docencia como en } \\
\text { los procesos formativos. }\end{array}$ \\
\hline $\begin{array}{l}\text { Valores profesionales y atribu- } \\
\text { tos éticos de la docencia }\end{array}$ & $\begin{array}{l}\text { Principios éticos que rigen } \\
\text { el actuar del docente en su } \\
\text { interacción con los estu- } \\
\text { diantes y en su práctica } \\
\text { académica cotidiana. }\end{array}$ & $\begin{array}{l}\text { 2) Principios personales- profesionales: Son una } \\
\text { serie de principios mínimos necesarios para el } \\
\text { logro del ethos profesional de la docencia, tales } \\
\text { como: Respeto hacia el estudiante, igualdad en } \\
\text { el trato, honestidad, tolerancia, escucha activa, } \\
\text { diálogo, justicia social, equidad y sentido hu- } \\
\text { mano. }\end{array}$ \\
\hline \multirow[t]{2}{*}{ Prácticas docentes éticas } & $\begin{array}{l}\text { Acciones y/o situaciones de } \\
\text { enseñanza y aprendizaje } \\
\text { donde se cristaliza la ética } \\
\text { profesional del docente uni- } \\
\text { versitario (interacción }\end{array}$ & $\begin{array}{l}\text { 3) Compromiso ético con la docencia: Se refiere a la } \\
\text { aceptación consciente de la labor docente que } \\
\text { implica el reconocimiento del bien común que } \\
\text { caracteriza el actuar ético en la enseñanza. }\end{array}$ \\
\hline & $\begin{array}{l}\text { profesor-estudiantes). } \\
\text { rignifica identificarse con el } \\
\text { aspectos que inciden en el } \\
\text { aprendiz. }\end{array}$ & $\begin{array}{l}\text { 4) Responsabilidad con la formación del estudiante: } \\
\text { Son las buenas prácticas docentes encamina- } \\
\text { das al beneficio y bienestar de los estudiantes, } \\
\text { evitando daño físico, moral o legal, cumpliendo } \\
\text { con las obligaciones docentes para la formación } \\
\text { estudiantil, demostrando con ello, maestría en } \\
\text { la práctica de la enseñanza. }\end{array}$ \\
\hline
\end{tabular}


Evaluación de la ética profesional docente
Acciones orientadas hacia la evaluación del desempeño ético del docente que pueden ser desarrolladas como parte de una estrategia implementada por la propia institución educativa.
5) La ética profesional como componente evaluativo: Se refiere a la presencia de características éticas como elementos evaluativos en el desempeño del docente universitario, así como la relevancia de la ética profesional docente en el marco normativo de la universidad.

6) Medios y procesos de evaluación de la ética docente: Son los recursos utilizados para evaluar el desempeño ético, así como el desarrollo de acciones concretas planificadas por la institución para evaluar el desempeño ético del docente universitario.

Nota. Elaboración propia.

El diseño del cuestionario asumió una escala Likert que, como señala Namakforoosh (2000), está constituida por una serie de reactivos ante los cuales se solicita la reacción del sujeto. La escala Likert como método de recopilación de datos es altamente reconocida en las ciencias sociales y humanas, cuyos fenómenos de investigación los constituyen los seres humanos, quienes aportan información valiosa o testimonios sobre el objeto de estudio (Fabila et al., 2013).

El cuestionario-escala tiene como propósito conocer la opinión de los docentes universitarios sobre aquellos aspectos que a su juicio caracterizan mejor la ética profesional y su evaluación en el marco del desempeño docente. La relevancia del instrumento radica en contar con información que permita caracterizar la ética profesional en la docencia en el contexto de la Universidad Autónoma del Estado de Hidalgo (UAEH), México.

\subsection{El proceso de validación del cuestionario}

La validación se realizó a través de una entrevista cognitiva, la cual constituye un excelente método para determinar la validez de un instrumento, ofreciendo como principal ventaja, la posibilidad de pensar en voz alta y hacer un sondeo verbal sobre la pertinencia de las preguntas de un instrumento, en este caso, el cuestionario.

De acuerdo con el Instituto William Davidson de la Universidad de Michigan (2019), los propósitos fundamentales que esta técnica ofrece son valorar la comprensión de las preguntas, es decir, si las preguntas de un instrumento reúnen la información deseada, evaluar que los encuestados entiendan cada pregunta de manera consistente y profundizar en cada pregunta para comprender las posibles reacciones e interpretación que el encuestado da a cada aseveración.

En la validación del cuestionario se siguió la metodología para la entrevista cognitiva propuesta por Willis (1999), la cual precisa iniciar por informar a los participantes sobre la naturaleza de la entrevista, los objetivos, establecer el espacio para la entrevista, garantizar la confidencialidad, respetar el tiempo del participante, tener tacto en el planteamiento de las preguntas, mostrar disposición y una escucha activa.

Como parte del proceso de validación de la entrevista, se tomaron en cuenta los siguientes lineamientos: comprensión de la pregunta, el proceso de respuesta, recuperación de información relevante y toma de decisiones en torno a las modificaciones en la redacción y uso de terminología de cada una de las preguntas, al igual que la pertinencia de las preguntas y su congruencia dentro de las dimensiones correspondientes.

De esta manera, se contó con la participación de seis académicos de distintas universidades mexicanas en la validación del cuestionario "Evaluación de la ética profesional de la docencia universitaria". De ellos, 3 fueron hombres y 3 mujeres; 3 laboran en universidades públicas y 3 en universidades privadas. Los académicos participantes laboran en universidades ubicadas en Oaxaca, Oaxaca; Colima, Colima; Mérida, Yucatán; Guadalajara, Jalisco; Ciudad de México y Pachuca, Hidalgo. En el Cuadro 3 se presentan los datos de los académicos participantes. Cabe señalar que los académicos no se conocían entre sí.

La entrevista cognitiva se realizó en forma individual durante el mes de julio de 2020. Se tuvo un primer contacto vía correo electrónico para solicitar su participación en la validación del instrumento. Una vez confirmada su participación, se procedió a comentar la dinámica de la entrevista cognitiva, se envió el cuestionario, sus objetivos e intención del estudio. Se estableció un tiempo de revisión del instrumento y envío de las observaciones.

Posteriormente se acordó una reunión vía remota con cada uno de ellos para hacer la entrevista cognitiva. La duración de la entrevista osciló entre 1 y 2 horas aproximadamente. 


\section{Resultados}

A partir de la revisión al instrumento en su forma escrita como de la entrevista cognitiva se obtuvieron las siguientes observaciones al instrumento:

- Mayor precisión en las instrucciones.

- Reducir el número de reactivos (todos sugirieron presentar un cuestionario más corto).

- Agregar más datos de los profesores en la ficha de identificación.

- Eliminar algunos reactivos confusos y repetitivos, cambiar términos y/o redacción en algunos de los reactivos.

- Cambiar los verbos en algunos de los reactivos (poner verbos en infinitivo).

- Considerar la cuestión de género (referirse a las y los estudiantes).

- Cambiar las opciones de respuestas en una dimensión.

- Equilibrar el número de ítems por dimensión, dejar más en la tercera y cuarta dimensión.

Cuadro 3

Datos de los participantes en la validación del cuestionario

\begin{tabular}{|c|c|c|c|c|c|c|c|c|}
\hline \multirow[b]{2}{*}{ Experto } & \multicolumn{2}{|c|}{ Género } & \multirow[b]{2}{*}{ Edad } & \multirow{2}{*}{$\begin{array}{l}\text { Formación } \\
\text { disciplinar }\end{array}$} & \multirow[b]{2}{*}{ Posgrado } & \multirow{2}{*}{$\begin{array}{l}\text { Universidad } \\
\text { donde labora }\end{array}$} & \multirow{2}{*}{$\begin{array}{c}\text { Tipo de } \\
\text { universidad }\end{array}$} & \multirow{2}{*}{$\begin{array}{c}\text { Experiencia } \\
\text { docente en } \\
\text { el nivel de } \\
\text { licenciatura }\end{array}$} \\
\hline & $\mathbf{M}$ & $\mathbf{F}$ & & & & & & \\
\hline Docente 1 & $\mathrm{X}$ & & 35 & $\begin{array}{l}\text { Ingeniería } \\
\text { en diseño. } \\
\text { Licenciatura } \\
\text { en pedago- } \\
\text { gía }\end{array}$ & $\begin{array}{l}\text { Maestría en diseño } \\
\text { de proyectos } \\
\text { educativos virtuales. } \\
\text { Maestría en genera- } \\
\text { ción y gestión de la } \\
\text { innovación }\end{array}$ & $\begin{array}{l}\text { Universidad } \\
\text { José Vasconce- } \\
\text { los, Oaxaca }\end{array}$ & $\begin{array}{c}\text { Privada } \\
\text { Cd. Oaxaca }\end{array}$ & 12 años \\
\hline Docente 2 & & $\mathrm{X}$ & 42 & $\begin{array}{c}\text { Licenciatura } \\
\text { en lengua } \\
\text { inglesa }\end{array}$ & $\begin{array}{l}\text { Maestría en ense- } \\
\text { ñanza del inglés. } \\
\text { Maestría en } \\
\text { educación }\end{array}$ & $\begin{array}{l}\text { Universidad } \\
\text { de Colima, } \\
\text { Colima. }\end{array}$ & $\begin{array}{c}\text { Pública } \\
\text { Cd. Colima }\end{array}$ & 17 años \\
\hline Docente 3 & & $\mathrm{X}$ & 37 & $\begin{array}{l}\text { Licencia- } \\
\text { tura en } \\
\text { educación } \\
\text { indígena }\end{array}$ & $\begin{array}{c}\text { Maestría en } \\
\text { pedagogía }\end{array}$ & $\begin{array}{l}\text { Universidad } \\
\text { Pedagógica } \\
\text { Nacional Uni- } \\
\text { dad 31-A } \\
\text { Yucatán }\end{array}$ & $\begin{array}{l}\text { Pública } \\
\text { Mérida, } \\
\text { Yucatán }\end{array}$ & 12 años \\
\hline Docente 4 & & $\mathrm{X}$ & 33 & $\begin{array}{l}\text { Licenciatura } \\
\text { en psicolo- } \\
\text { gía }\end{array}$ & $\begin{array}{l}\text { Maestría en política } \\
\text { y gestión pública }\end{array}$ & $\begin{array}{l}\text { ITESO } \\
\text { Jalisco }\end{array}$ & $\begin{array}{l}\text { Privada } \\
\text { Guadalaja- } \\
\text { ra, Jalisco }\end{array}$ & 6 años \\
\hline Docente 5 & $\mathrm{X}$ & & 47 & $\begin{array}{l}\text { Licenciatura } \\
\text { en derecho }\end{array}$ & $\begin{array}{c}\text { Maestría en ad- } \\
\text { ministración de la } \\
\text { justicia y derechos } \\
\text { humanos }\end{array}$ & $\begin{array}{l}\text { Lasalle y Tec- } \\
\text { nológico de } \\
\text { Monterrey } \\
\text { Hidalgo }\end{array}$ & $\begin{array}{l}\text { Privadas } \\
\text { Pachuca, } \\
\text { Hidalgo }\end{array}$ & 23 años \\
\hline Docente 6 & $\mathrm{X}$ & & 58 & $\begin{array}{l}\text { Licenciatura } \\
\text { en psicolo- } \\
\text { gía }\end{array}$ & $\begin{array}{l}\text { Máster en didáctica } \\
\text { de las matemáticas }\end{array}$ & $\begin{array}{l}\text { Universidad } \\
\text { Pedagógica } \\
\text { Nacional Uni- } \\
\text { dad Ajusco, } \\
\text { Ciudad de } \\
\text { México }\end{array}$ & $\begin{array}{l}\text { Pública } \\
\text { Ciudad de } \\
\text { México }\end{array}$ & 31 años \\
\hline
\end{tabular}

Nota. Elaboración propia. 
Todos los académicos estuvieron de acuerdo en la pertinencia de los reactivos como parte descriptiva de la ética profesional de la docencia universitaria en las interacciones entre profesores y alumnos, así como de la relevancia de la evaluación del desempeño del profesor universitario desde su dimensión ética. A partir de las observaciones realizadas, se realizaron los ajustes al cuestionario atendiendo a las observaciones donde hubo un consenso. El cuestionario de inicio tenía 76 reactivos, una vez con los ajustes realizados quedaron un total de 49 ítems. El cuestionario se encuentra estructurado en 4 dimensiones, la primera dimensión "la ética en la docencia universitaria" está conformada por 6 reactivos; la segunda dimensión "Valores profesionales y atributos éticos de la docencia" compuesta por 11 reactivos; la tercera dimensión "Prácticas docentes éticas" constituida por 20 reactivos y la cuarta dimensión "Evaluación de la ética profesional docente" con 12 reactivos. En las dimensiones 3 y 4 los reactivos están clasificados en dos ejes analíticos respectivamente. Dicho instrumento en su versión final aparece en el Cuadro 4.

En cuanto a los datos de la ficha de identificación docente, se determinaron los siguientes aspectos:

- Datos personales: edad, género, estado civil.

- Formación profesional: formación disciplinar, posgrados, cursos de formación docente sobre ética.

- Experiencia docente: años de servicio en la docencia en el nivel superior y antigüedad en la universidad.

- Situación laboral: dedicación exclusiva a la docencia, ejercicio de la propia profesión, tipo de contratación, pertenencia al Sistema Nacional de Investigadores (SNI).

- Docencia: programas en los que ejerce la docencia y promedio de estudiantes atendidos durante el semestre.

El cuestionario así diseñado permitirá contar con un primer acercamiento a la caracterización de la ética profesional en la docencia universitaria y su delimitación en los procesos de evaluación del desempeño docente.

Cuadro 4

Cuestionario de evaluación de la ética profesional docente por dimensión, ejes analíticos y tipo de escala

\begin{tabular}{|c|c|c|c|}
\hline Dimensiones & $\begin{array}{c}\text { Ejes } \\
\text { analíticos }\end{array}$ & Ítems & $\begin{array}{l}\text { Escala de } \\
\text { respuesta }\end{array}$ \\
\hline $\begin{array}{l}\text { La ética en } \\
\text { la docencia } \\
\text { universitaria }\end{array}$ & $\begin{array}{l}\text { La pro- } \\
\text { fesión } \\
\text { docente }\end{array}$ & $\begin{array}{l}\text { 1. Me identifico con la profesión docente } \\
\text { 2. Tengo vocación por la enseñanza } \\
\text { 3. Me siento motivado al desempeñarme como docente } \\
\text { 4. Disfruto las actividades de la docencia } \\
\text { 5. Me identifico con los valores de la institución } \\
\text { 6. Me capacito en temas sobre ética para ser un mejor docente }\end{array}$ & $\begin{array}{l}\text { 1. Totalmente } \\
\text { en desacuerdo } \\
\text { 2. En desacuerdo } \\
\text { 3. De acuerdo } \\
\text { 4. Totalmente } \\
\text { de acuerdo }\end{array}$ \\
\hline $\begin{array}{l}\text { Valores } \\
\text { profesionales } \\
\text { y atributos } \\
\text { éticos de la } \\
\text { docencia }\end{array}$ & $\begin{array}{l}\text { Principios } \\
\text { personales- } \\
\text { profesio- } \\
\text { nales }\end{array}$ & $\begin{array}{l}\text { 7. Tratar con cordialidad y amabilidad a los y las estudiantes } \\
\text { 8. Demostrar empatía ante las necesidades de los y las estudian- } \\
\text { tes } \\
\text { 9. Comunicarse asertivamente con los y las estudiantes } \\
\text { 10. Brindar confianza a los y las estudiantes para que externen } \\
\text { sus opiniones } \\
\text { 11. Actuar con paciencia ante las dificultades de los y las estu- } \\
\text { diantes } \\
\text { 12. Brindar un trato equitativo a los y las estudiantes } \\
\text { 13. Mostrar interés genuino por el bienestar de los y las estudian- } \\
\text { tes en su proceso formativo dentro de la universidad } \\
\text { 14. Respetar las creencias y opiniones de los y las estudiantes } \\
\text { 15. Respetar las tradiciones culturales y lingüísticas de los estu- } \\
\text { diantes } \\
\text { 16. Promover en los y las estudiantes la superación personal y el } \\
\text { deseo de aprender } \\
\text { 17. Promover en los y las estudiantes el amor a sí mismos }\end{array}$ & $\begin{array}{l}\text { 1. No es impor- } \\
\text { tante } \\
\text { 2. Poco impor- } \\
\text { tante } \\
\text { 3. Importante } \\
\text { 4. Muy impor- } \\
\text { tante }\end{array}$ \\
\hline
\end{tabular}




\begin{tabular}{|c|c|c|c|}
\hline \multirow{20}{*}{$\begin{array}{l}\text { Prácticas } \\
\text { docentes } \\
\text { éticas }\end{array}$} & \multirow{11}{*}{$\begin{array}{l}\text { Compromi- } \\
\text { so ético con } \\
\text { la docencia }\end{array}$} & 18. Respetar los horarios de entrada y salida de clase & \multirow{11}{*}{$\begin{array}{l}\text { 1. No es impor- } \\
\text { tante } \\
\text { 2. Poco impor- } \\
\text { tante } \\
\text { 3. Importante } \\
\text { 4. Muy impor- } \\
\text { tante }\end{array}$} \\
\hline & & $\begin{array}{l}\text { 19. Establecer los criterios de evaluación de los aprendizajes en } \\
\text { conjunto con el grupo }\end{array}$ & \\
\hline & & $\begin{array}{l}\text { 20. Evaluar el aprendizaje estudiantil con apego a los lineamien- } \\
\text { tos establecidos con el grupo }\end{array}$ & \\
\hline & & $\begin{array}{l}\text { 21. Cuidar que los puntajes de evaluación de los aprendizajes } \\
\text { sean congruentes con el nivel de complejidad de los contenidos } \\
\text { educativos }\end{array}$ & \\
\hline & & $\begin{array}{l}\text { 22. Establecer las normas de clase en acuerdo con los y las estu- } \\
\text { diantes }\end{array}$ & \\
\hline & & 23. Propiciar un clima de armonía en las clases & \\
\hline & & 24. Evitar cualquier forma de discriminación en el aula & \\
\hline & & 25. Evitar comentarios o chistes con contenido sexual o agresivos & \\
\hline & & $\begin{array}{l}\text { 26. Generar un entorno educativo que promueva en los y las } \\
\text { estudiantes la vocación hacia el ejercicio de su profesión }\end{array}$ & \\
\hline & & 27. Demostrar sinceridad en el discurso académico & \\
\hline & & $\begin{array}{l}\text { 28. Reflexionar ante los dilemas éticos que plantea la práctica } \\
\text { docente }\end{array}$ & \\
\hline & \multirow{9}{*}{$\begin{array}{l}\text { Responsa- } \\
\text { bilidad con } \\
\text { la forma- } \\
\text { ción del } \\
\text { estudiante }\end{array}$} & 29. Promover el espíritu crítico en el estudiante & \multirow{9}{*}{$\begin{array}{l}\text { 1. No es impor- } \\
\text { tante } \\
\text { 2. Poco impor- } \\
\text { tante } \\
\text { 3. Importante } \\
\text { 4. Muy impor- } \\
\text { tante }\end{array}$} \\
\hline & & $\begin{array}{l}\text { 30. Discutir con los y las estudiantes sobre problemáticas socia- } \\
\text { les }\end{array}$ & \\
\hline & & $\begin{array}{l}\text { 31. Brindar las mismas oportunidades de aprendizaje al colectivo } \\
\text { de los y las estudiantes }\end{array}$ & \\
\hline & & $\begin{array}{l}\text { 32. Esforzarse por diseñar experiencias que favorezcan aprendi- } \\
\text { zajes significativos }\end{array}$ & \\
\hline & & $\begin{array}{l}\text { 33. Realizar adaptaciones a las actividades de enseñanza y de } \\
\text { aprendizaje en función de los intereses de los y las estudiantes }\end{array}$ & \\
\hline & & $\begin{array}{l}\text { 34. Brindar retroalimentación a los y las estudiantes a partir de } \\
\text { la presentación de sus resultados de evaluación }\end{array}$ & \\
\hline & & $\begin{array}{l}\text { 35. Reconocer el esfuerzo de los y las estudiantes diciéndoles lo } \\
\text { que hacen bien }\end{array}$ & \\
\hline & & $\begin{array}{l}\text { 36. Orientar a los y las estudiantes cuando solicitan opinión } \\
\text { académica }\end{array}$ & \\
\hline & & 37. Brindar asesoría individual ante dificultades de aprendizaje & \\
\hline \multirow{12}{*}{$\begin{array}{l}\text { Evaluación } \\
\text { de la ética } \\
\text { profesional } \\
\text { docente }\end{array}$} & \multirow{6}{*}{$\begin{array}{l}\text { La ética } \\
\text { profesional } \\
\text { como com- } \\
\text { ponente } \\
\text { evaluativo }\end{array}$} & $\begin{array}{l}\text { 38. Tomar en cuenta la opinión estudiantil respecto al desempe- } \\
\text { ño para planear mejoras en la docencia }\end{array}$ & \multirow{6}{*}{$\begin{array}{l}\text { 1. No es relevante } \\
\text { 2. Poco relevante } \\
\text { 3. Relevante } \\
\text { 4. Muy relevante }\end{array}$} \\
\hline & & $\begin{array}{l}\text { 39. Identificar las debilidades y fortalezas docentes a partir de } \\
\text { los resultados de evaluación docente institucional }\end{array}$ & \\
\hline & & $\begin{array}{l}\text { 40. Retroalimentar la práctica docente a partir de los resultados } \\
\text { del aprendizaje de los estudiantes }\end{array}$ & \\
\hline & & $\begin{array}{l}\text { 41. Retroalimentar el desempeño docente didáctica y pedagógi- } \\
\text { camente a partir del diálogo entre docentes y estudiantes }\end{array}$ & \\
\hline & & $\begin{array}{l}\text { 42. Retroalimentar el desempeño docente didáctica y pedagógi- } \\
\text { camente a partir del diálogo entre colegas }\end{array}$ & \\
\hline & & $\begin{array}{l}\text { 43. Promover en los docentes el análisis crítico de resultados de } \\
\text { su propio desempeño docente }\end{array}$ & \\
\hline & \multirow{6}{*}{$\begin{array}{l}\text { Medios y } \\
\text { procesos de } \\
\text { evaluación } \\
\text { de la ética } \\
\text { docente }\end{array}$} & $\begin{array}{l}\text { 44. Atender a las debilidades docentes brindando capacitación } \\
\text { continua y/o ayuda }\end{array}$ & \multirow{6}{*}{$\begin{array}{l}\text { 1. No es relevante } \\
\text { 2. Poco relevante } \\
\text { 3. Relevante } \\
\text { 4. Muy relevante }\end{array}$} \\
\hline & & 45. Propiciar la reflexión sobre el desempeño ético en la docencia & \\
\hline & & 46. Reconocer el buen desempeño en el ejercicio de la docencia & \\
\hline & & $\begin{array}{l}\text { 47. Explicitar el impacto de la labor docente en la formación de } \\
\text { cuadros profesionales }\end{array}$ & \\
\hline & & $\begin{array}{l}\text { 48. Diseñar instrumentos para evaluar la ética profesional do- } \\
\text { cente }\end{array}$ & \\
\hline & & $\begin{array}{l}\text { 49. Desarrollar procesos institucionales para evaluar la ética en } \\
\text { el desempeño docente }\end{array}$ & \\
\hline
\end{tabular}

Nota. Elaboración propia. 


\section{Discusión y conclusiones}

El proceso de validación del instrumento a través de la entrevista cognitiva resultó una estrategia metodológica muy enriquecedora en la definición del cuestionario escala, ya que la participación crítica de los académicos participantes de distintas universidades del país permitió situar la discusión sobre la pertinencia de una serie de rasgos, actitudes y prácticas que caracterizan la ética profesional en situaciones de enseñanza-aprendizaje entre docentes y estudiantes. Las aportaciones de los académicos demostraron la posibilidad de hacer visible la ética profesional en los procesos de interacción cotidiana entre docentes y estudiantes, además de la relevancia de la ética como base medular de una docencia de excelencia.

De igual manera, fue posible identificar los diferentes medios y procesos que a juicio de los expertos son elementos clave en la evaluación de la ética profesional docente, actitudes generalmente implícitas en los instrumentos de evaluación del desempeño docente en la actualidad. Reconocer la necesidad de evaluar el desempeño ético del docente universitario es una oportunidad para posicionar a la docencia como una profesión humana, cuya principal tarea está orientada a favorecer el desarrollo integral de los futuros profesionistas.

El diseño del cuestionario aquí presentado pretende contribuir a la necesidad de reconocer a la ética profesional como un eje que subyace al profesionalismo en la docencia universitaria, ya que como señalan Sanz (2019), Hirsch (2019), Chávez (2019), Camps (2008) y Chávez y Benavides (2011) en las prácticas de enseñanza y aprendizaje es donde los profesores maniestan una serie de actitudes y acciones éticas, demostrando con ello su compromiso con la docencia y con la formación integral de los estudiantes universitarios. De ahí la relevancia que tiene la opinión docente en la construcción de instrumentos y/o procesos de evaluación centradas en la ética profesional de la docencia.

También se busca dar cuenta de la generación de nuevas dimensiones de valoración del desempeño docente que delimiten el actuar ético en la docencia y con ello, proponer una serie de características éticas presentes en las prácticas cotidianas docentes en aras de favorecer una evaluación de la docencia integral.

La relevancia del cuestionario será visibilizar aquellas actitudes, acciones y situaciones que desde la perspectiva de los propios docentes están presentes en sus interacciones cotidianas con los estudiantes y que pueden ser reconocidos como rasgos que caracterizan la ética profesional en la docencia en el nivel superior, con lo cual se podrían generar procesos de evaluación docente orientados al mejoramiento del profesor desde su dimensión humana (Loredo, 2000; Arbesú, et. al. 2011).

Desde una perspectiva de la evaluación formativa, el cuestionario podría dar lugar a procesos de autoevaluación del desempeño docente, generando espacios de reflexión y autocrítica sobre la ética profesional en el ejercicio de la docencia, dando lugar a procesos de concientización, propiciando con ello, cambios que lleven a los profesores a sumir un compromiso ético con la docencia.

La validación del instrumento de evaluación de la ética profesional docente constituye la primera fase de una investigación más amplia que contempla otros procesos metodológicos de carácter cualitativo donde se busca profundizar en las experiencias docentes en torno a la evaluación de la ética profesional en la docencia en el nivel superior.

\section{Referencias}

Águila, M. M. J. B., Croda, B. P. e Iturbide, F. P. (2014). Ética profesional de la docencia, desde la perspectiva de estudiants de la maestría en pedagogía. En J. M. López-Calva y M. L. Gaeta (Coords.), Ética y autorregulación en la formación de profesionales de la educación (pp. 25-47). Ibero-UPAEP.

Álvarez-Gayou, J. J. L. (2019). Cómo hacer investigación cualitativa. Fundamentos y metodología. Paidós educador.

Arbesú, M. I., Canales, A., Crispín, M. L., Cruz, I., Figueroa, A. E. y Gilio, M. C. (2011). Las políticas y los usos de la evaluación de la docencia en la educación superior: Planteamientos y perspectivas. En M. Rueda y F. Díaz-Barriga (Coords.), La evaluación de la docencia en la universidad. Perspectivas desde la investigación y la intervención profesional (pp. 203-252). IISUE, UNAM. 
Campo-Cabal, G. (2012). Conductas transgresoras en el ámbito académico. Revista Colombiana de Psiquiatría, 41, 120-135.

Camps, V. (1996). Virtudes públicas. Espasa-Calpe.

Camps, V. (2008). Creer en la educación. La asignatura pendiente. Península Atalaya.

Chávez, G. G. (2019). Conocimiento, didáctica y empatía: rasgos de los profesores de excelencia según los estudiantes universitarios. En A. A. Hirsch y J. Pérez-Castro (Coords.), Ética profesional y responsabilidad social universitaria: experiencias institucionales (pp.149-164). IISUE Educación.

Chávez, G. G. y Benavides, M. B. (2011). Los profesores universitarios: entre la exigencia profesional y el compromiso ético-social. Sinéctica: Revista Electrónica de Educación, 37, 1-13.

Coloma, M. C. R. (2010). Estudio comprensivo sobre la evaluación del docente. Revista Iberoamericana de Evaluación Educativa, 3(1e), 61-76.

Cortina, A. (2017). ¿Para qué sirve realmente...? La ética. Paidós.

Díaz-Barriga, A. F., Peréz, R. M. A. y Lara, G. Y. (2016). Para enseñar ética profesional no basta con una asignatura: Los estudiantes de psicología reportan incidentes críticos en aulas y en escenarios reales. Universia, 8(18), 4258 .

Fabila, E. A. M., Minami, H. e Izquierdo, S. M. J. (2013). La Escala Likert en la evaluación docente: acercamiento a sus características y principios metodológicos. Perspectivas Docentes, 50, 31-40.

Finkler, M., Caetano, J. C. y Sousa, R. F. R. (2012). Ethical-pedagogical care in the process of professional socialization: towards ethical education. Interface-Comunicaçao, 16(43), 981-993.

Flick, U. (2014). The SAGE Handbook of qualitative data analisis. SAGE.

García-Cabrero, B., Loredo, E. J., Luna, S. E. y Rueda, B. M. (2014). Competencias docentes en educación mediay superior. Desarrollo y validación de un modelo de evaluación. Juan Pablos Editor.

García, R., Ferrández, R., Sales, M. A. y Moliner, M. O. (2006). Elaboración de instrumentos de medida de las actitudes y opiniones del profesorado universitario hacia la ética profesional docente y su papel como transmisor de valores. Revista Electrónica de Investigación y Evaluación Educativa, 12(1), 129-149.

García, R., Jover, G. y Escámez, S. J. (2010). Ética profesional docente. Síntesis.

García, L. R., Verde, P. I. y Vázquez, V. (2011, octubre). ¿Por qué es necesario trabajar la dimensión ética en la docencia? Comunicación presentada en el XII Congreso Internacional de Teoría de la Educación, Universitat de Barcelona, España.

Gómez, C. (2014). Doce textos fundamentales de la ética del siglo XX. Alianza.

Hirsch, A. A. (2010a). Formación en ética profesional y los profesores de posgrado de la Universidad Nacional Autónoma de México. Revista Electrónica de Investigación Educativa, 12(e).

Hirsch, A. A. (2010b). Ética profesional y profesores universitarios: Una perspectiva comparada. Revista Reencuentro, $57,33-38$

Hirsch, A. A. (2011). Dilemas, tensiones y contradicciones en la conducta ética de los profesores. Sinéctica: Revista Electrónica de Educación, 37, 1-16.

Hirsch, A. A. (2019). Dimensiones y rasgos sobre la excelencia del profesorado de posgrado de la UNAM. En A. A. Hirsch y J. Pérez-Castro (Coords.), Ética profesional y responsabilidad social universitaria: experiencias institucionales (pp. 133-148). IISUE Educación.

Hirsch, A. A. y Yurén, T. (2013). La investigación en México en el campo educativo y valores 2002-2011. ANUIES-COMIE.

Hortal, A. (2000). Docencia. En A. A. Hirsch y J. Pérez-Castro (Coords.), Ética profesional y responsabilidad social universitaria: experiencias institucionales (pp. 94-97). IISUE Educación.

Hortal, A. (2002). Ética general de las profesiones. Desclée De Brouwer. 
Iglesias, S. M. M. y Loredo, E. J. (2017, noviembre). La evaluación de la docencia en instituciones de educación superior: Significado para el docente. Ponencia presentada en el XIV Congreso Nacional de Investigación Educativa COMIE. Universidad Autónoma de San Luis Potosí, México.

Jaramillo, E. L. G. y Aguirre, G. J. C. (2010). Lévinas y las ciencias sociales: Fundamentos epistémicos desde la alteridad. Revista Folios, 31, 3-19.

Lévinas, E. (2001). La huella del otro. Taurus.

López-Calva, J. M., Benítez, U. M. P. y Méndez, E. C. R. (2017, noviembre). ¿Educación para la renta o para la democracia? Rasgos de un buen profesional desde la visión de estudiantes y profesores de una universidad privada. Ponencia presentada en el XIV Congreso Nacional de Investigación Educativa COMIE. Universidad Autónoma de San Luis Potosí, México.

López-Calva, J. M. y Gómez, V. M. I. P. (2017, noviembre). Valores profesionales y excelencia docente desde la perspectiva de los profesores de una universidad privada. Ponencia presentada en el XIV Congreso Nacional de Investigación Educativa COMIE. Universidad Autónoma de San Luis Potosí, México.

Loredo, E. J. (2000). Evaluación de la práctica docente en educación superior. Porrúa.

Mayorga, K. (2018). Igualdad de género en la educación superior en el siglo XXI. Palermo Business Review, 18, 137-144.

Medina, R. A., Domínguez, G, M. C. y Medina, D. M. (2010). Teaching competencies assessment. Innovacion Educativa, 10(53), 73-95.

Murillo, F. J. (2018, septiembre). Una evaluación docente para la justicia social. Conferencia inaugural en el Congreso Internacional de Educación Evaluación 2018. Universidad Autónoma de Tlaxcala, México.

Murillo, F. J., Román, M. y Hernández-Castilla, R. (2011). Evaluación educativa para la justicia social. Revista Iberoamericana de Evaluación Educativa, 4(1), 1-17.

Namakforoosh, M. (2000). Metodología de la investigación. Limusa.

Nava, P. J. M. (29 de octubre de 2011). Falta de formación ética en el profesorado. Entrevista realizada por Sepúlveda, V. L. Gaceta Universitaria, sector “Educación”. UNAM.

Navia, A. C. (2019). Aproximaciones conceptuales en torno al buen docente en educación superior. En A. A. Hirsch y J. Pérez-Castro (Coords.), Ética profesional y responsabilidad social universitaria: Experiencias institucionales (pp. 181-195). IISUE Educación.

Rancich, A. M., Donato, M. y Gelpi, R. J. (2015). Relación docente-alumno: Percepción de incidentes moralmente incorrectos. Persona y Bioética, 19(2), 319-329. https://doi.org/10.5294/pebI.2015.19.2.10

Rueda, B. M. (2010). Reflexiones generales a considerar en el diseño y puesta en operación de programas de evaluación de la docencia. Revista Revista Iberoamericana de Evaluación Educativa, 3(1e), 345-351.

Ruiz, R. V. (2016). Ética y mundo actual. Universidad Iberoamericana.

Safari, Y. y Yoosefpour, N. (2018). Dataset for assessing the professional ethics of teaching by medical teachers from the perspective of students in Kermanshah Universtity of Medical Sciencies, Iran. Data in Brief 20, 1955-1959. https://doi.org/10.1016/j.dib.2018.09.060

Salazar, A. J., Inostroza, H. P., Marín, A. P., Gutiérrez, T. M. y Olivera, A. L. (2018). Modelo de evaluación docente. Una experiencia endógena como alternativa a la realidad de los profesores chileno. Edetania, 54, 93-105.

Sanz, P. R. (2019). Ética profesional y evaluación. Un análisis de las buenas prácticas docentes. En A. A. Hirsch, y J. Pérez-Castro (Coords.), Ética profesional y responsabilidad social universitaria: Experiencias institucionales (pp. 79 99). IISUE Educación.

Serrano, Z., Osses, L. M. y Molina, V. (2018). Evaluación y desarrollo de competencias éticas de docentes en servicio. Universidad de Chile.

Solano, G. W. y Campos, C. J. (2014). Hacia la construcción de un perfil para el docente del futuro. Innovaciones Educativas, 21, 85-107. 
Tapia, G. A. (2019). Concepciones y criterios de valoración del profesorado universitario perteneciente al ámbito de las humanidades sobre la relación entre ética y excelencia docente. Praxis Sociológica, 24, 201-212.

Torres, M. R. (2006). El profesor universitario, un agente de desarrollo moral. Revista Ciencias de la Salud, 4(e), 103109.

UNESCO. (2016). Guía para la igualdad de género en las políticas y prácticas de la formación docente. UNESCO.

Villarroel, V. A. y Bruna, D. V. (2017). Competencias pedagógicas que caracterizan a un docente universitario de excelencia: Un estudio de caso que incorpora la perspectiva de docentes y estudiantes. Formación Universitaria, 10(4), 75-96. https://doi.org/10.4067/S0718-50062017000400008

William Davidson Institute at the University of Michigan. (2019). Cognitive interview guide developed by the performance measurement initiative. American Statistical Association.

Willis, G. B. (1999). Cognitive interviewing. A “how to" guide. American Statistical Association.

\section{Breve CV de los autores}

\section{Alma Delia Torquemada González}

Licenciatura en Psicología Educativa, Universidad Pedagógica Nacional (UPN); Maestría en Psicología Educativa, Facultad de Psicología, UNAM. Estudiante del Doctorado Interinstitucional en Educación, Universidad Iberoamericana, Ciudad de México. Profesora investigadora del Área Académica de Ciencias de la Educación, Instituto de Ciencias Sociales y Humanidades, Universidad Autónoma del Estado de Hidalgo, México. Docente en nivel de licenciatura y maestría en ciencias de educación y especialidad en docencia. Líneas de investigación: tutoría universitaria, evaluación docente y formación para la investigación. Participación en eventos académicos relacionados con la tutoría universitaria y evaluación docente en el nivel superior. Miembro de la Red Iberoamericana de Investigadores sobre Evaluación de la Docencia (RIIED). Email: almatorquemada@yahoo.com.mx

ORCID ID: https://orcid.org/0000-0003-2569-1787

\section{Javier Loredo Enríquez}

Doctorado en Ciencias de la Educación y Maestría en Educación Universidad René Descartes, Paris V, Sorbonne, Francia. Licenciatura en Pedagogía, Universidad Nacional Autónoma de México. Coordinador de posgrado, tanto de maestría como de doctorado en diversas universidades. Miembro del grupo que diseño y puso en marcha el Doctorado Interinstitucional en Educación de cuatro universidades jesuitas. Coordinador de la Maestría en Investigación y Desarrollo Educativo de la Universidad Iberoamericana, CDMX. Evaluador, consejero editorial y dictaminador en revistas nacionales e internacionales en educación. Evaluador de Programas inscritos en el Padrón Nacional de Programas de Calidad (PNPC) 2017. Evaluador de Estancias Posdoctorales que otorga CONACYT y ponente en congresos nacionales e internacionales. Líneas de investigación: Evaluación de la práctica docente, formación docente, diseño y evaluación del currículo. Tiene publicaciones tanto en libros, capítulos de libro y artículos en la línea de la evaluación de la práctica docente y currículo. Es miembro de la Red Iberoamericana de Investigadores sobre la Evaluación de la Docencia (RIIED). Email: javier.loredo@gmail.com

ORCID ID: https://orcid.org/0000-0002-0620-566X 